\title{
HPLC and HRGC Analyses of Polyphenols and Secoiridoid in Olive Oil
}

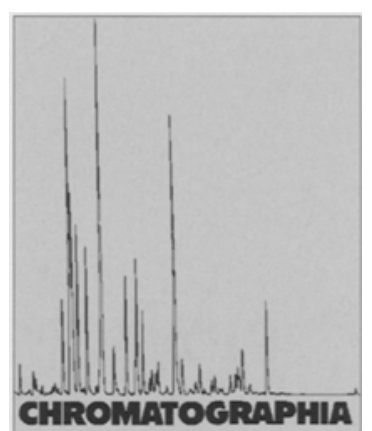

$2001,53,279-284$

\author{
A. Romani ${ }^{1 *}$ / P. Pinelli ${ }^{1}$ / N. Mulinacci / C. Galardi 1 / F. F. Vincieri / L. Liberatore ${ }^{2}$ / A. Cichelli \\ ${ }^{1}$ Dipartimento di Scienze Farmaceutiche, Università degli Studi di Firenze, Via G. Capponi 9, 50121 Firenze, Italy; \\ E-Mail: romani.a@farmfi.scifarm.unifi.it \\ 2 Dipartimento di Scienze, Università Chieti-Pescara, Viale Pindaro 42, 65127, Pescara, Italy
}

\section{Key Words}

Column liquid chromatography

Gas chromatography

Solid phase extraction

Secoiridoid derivatives

\section{Summary}

Phenolic compounds influence the sensorial properties of both olives and virgin oil and are important markers for studying the characteristics of the fruits and controlling virgin oil production processes. The aim of this investigation was to evaluate the polyphenolic and secoiridoid content of various virgin olive oils from Abruzzo (Italy) to obtain knowledge on quali-quantitative profiles of these compounds in samples obtained from the same harvesting season (1998). These oils were collected from the most frequent Abruzzo cultivars, Gentile, Leccino and Dritta, by two different processing techniques: a process of milling and continuous washing with water or by traditional press. A quali-quantitative analysis was performed by HPLC-DAD, HPLC-MS and HRGC to characterize the different subclasses, and in particular the following compounds were identified and calibrated: tyrosol, hydroxytyrosol, phenolic acids (ferulic, syringic, caffeic and p-coumaric acids), oleuropein aglycone, deacetoxyoleuropein aglycone, elenolic acid and derivatives, other secoiridoid compounds and flavone aglycons (luteolin and apigenin).

\section{Introduction}

The beneficial health properties of olive oil have been known for centuries, particularly in the Mediterranean region where olives and olive oil are an inherent part of the culture and diet. The decreased incidence of cardiovascular disease in this area has been attributed mainly to their consumption [1] and the beneficial effects have been related to the antioxidant composition, namely, tocopherols and phenolic compounds [2,3]. Numerous pharma- cological studies demonstrate that a polyphenolic extract of olive oil and olive mill waste waters may protect the LDL (Low Density Lipoproteins) against oxidative modifications [4-6]. Moreover, the incubation of platelets with hydroxytyrosol causes a significant decrease in platelet aggregation induced by collagen under conditions of oxidative stress [7]. From these results it appears evident that the phenolic compounds of virgin olive oil play an important role in human nutrition as preventive agents against several diseases.
These molecules influence the sensorial properties of both olives and virgin oil and are important markers for studying the characteristics of the fruits and for controlling the virgin oil production processes. The knowledge of the variations in phenolic compounds make it possible to obtain better understanding of the relationships that may exist between these substances and the physiology and organoleptic quality of the fruit, as well as to provide a more solid basis for olive storage conditions and processing techniques, thus leading to improved quality [8-11].

Moreover, the high stability of virgin olive oil against thermoxidation and autoxidation processes over time could be related to the tocopherol and polyphenol concentration. In fact the oxidative stability of virgin oils decreases considerably after elimination of the polyphenols and also seems to depend on the polyunsaturated fatty acid concentration [12]

The contribution of individual phenols to oil quality has not been fully investigated and there is still more to be known about the importance of phenolic components for the sensory quality and the stability of the oil. The polyphenolic content of olive oil can vary greatly depending on several factors: oil production process, type of cultivar, growing conditions and time of ripening.

In the last twenty years considerable efforts have been made toward developing analytical procedures (gas chromatographic and/or HPLC sometimes assisted by mass spectroscopy) in order to separate, identify and characterize, by NMR 
Table I. List of olive oil samples obtained from the main Abruzzo cultivars (Gentile, Leccino and Dritta) crushing the fruits by using a process of milling and continuous washing with water or by traditional press.

\begin{tabular}{|lll|}
\hline Cultivar & Location & Processing Techniques \\
\hline Gentile $\left(\mathrm{G} 1_{\mathrm{C}}\right)$ & Campli $(\mathrm{Te})$ & Continuous \\
Gentile $\left(\mathrm{G} 2_{\mathrm{C}}\right)$ & Casoli $(\mathrm{Ch})$ & Continuous \\
Gentile $\left(\mathrm{G} 3_{\mathrm{T}}\right)$ & Rocca $\mathrm{S}$. Giovanni $(\mathrm{Ch})$ & Traditional \\
Leccino $\left(\mathrm{L1} \mathrm{C}_{\mathrm{C}}\right)$ & Ortona $(\mathrm{Ch})$ & Continuous \\
Leccino $\left(\mathrm{L} 2_{\mathrm{T}}\right)$ & Rocca $\mathrm{S}$. Giovanni $(\mathrm{Ch})$ & Traditional \\
Dritta $\left(\mathrm{D} 1_{\mathrm{C}}\right)$ & Moscoufo $(\mathrm{Pe})$ & Continuous \\
Dritta $\left(\mathrm{D} 2_{\mathrm{C}}\right)$ & Loreto $(\mathrm{Pe})$ & Continuous \\
Dritta $\left(\mathrm{D} 3_{\mathrm{T}}\right)$ & Pianella $(\mathrm{Pe})$ & Traditional \\
\hline
\end{tabular}

techniques, as many compounds as possible from the polar fraction of olives and virgin olive oil [13-20]. In particular, an important component of the polar fraction of olive oil is represented by secoiridoids. These molecules belong to the same biogenetics class, having loganic acid as precursor and oleuropein is the wellknown compound. Oleuropein is part of a wide group of natural glucosides typical of the Oleaceae family, the "secoiridoid glucosides" [21]. These molecules are characterized by a monoterpenic ciclopentanoic skeleton with the hydroxyl in position 1 is linked to glucose.

Montedoro et al. [22] reported a wide range of total phenols for Italian oils and subdivided the oils into three groups based on total phenols (calculated as gallic acid), namely, low (50-200 ppm); medium $(200-500 \mathrm{ppm})$ and high $\left(500_{-}\right.$ $1000 \mathrm{ppm})$. The differences in magnitude of the phenol content depend mainly on the cultivar. Montedoro et al. [22, 23, 17] has extensively studied the composition of simple and hydrolyzable phenols in virgin olive oil in relation to its oxidative stability. In particular, they reported that phenolic substances, which were correlated with the stability of the oil, contained either hydroxytyrosol or tyrosol-elenolic moieties in their molecules.

Few studies have been performed until now on the quantification of the single polyphenols present in olive oils, and often the quantitative data are related to the total polyphenolic concentration or to the total of a specific subclass, such as the "simple polyphenols" (tyrosol, hydroxytyrosol and elenolic acid) [20].

Within the context of our work to develop an initial database on germplasm collection and to establish the criteria and indicators useful for correlating the agronomic characteristics with the quality of the relative olive oil, two chromatographic techniques, HPLC (High Performance Liquid Chromatography) and
HRGC (High Resolution Gas Chromatography) were applied to identify and calibrate different phenolic subclasses.

For this purpose several virgin olive oils from Abruzzo (Italy) were employed, to obtain knowledge about quali-quantitative profiles of these compounds, in samples collected from the same harvesting season, and processed using two different techniques, namely by a process of milling and continuous washing with water, or by traditional press.

\section{Experimental}

The olive fruits were harvested at technological ripening in November, and the relative oils were immediately obtained crushing the olives by continuous (C) or traditional $(\mathrm{T})$ processing techniques. The virgin oil samples, extracted from the olives of three different varieties cultivated in Abruzzo (Leccino, Gentile and Dritta), are reported in Table I.

\section{Sample Preparation}

\section{Extraction of Polyphenols}

A quantity of $100 \mathrm{~mL}$ of each oil sample was extracted with $300 \mathrm{~mL}$ of the following solution: $\mathrm{EtOH} / \mathrm{H}_{2} \mathrm{O} 70: 30 v / v$, the water was acidified with formic acid $(\mathrm{pH}=2.5)$ and added to $1 \mathrm{~mL}$ of gallic acid solution (internal standard; EtOH/ $\mathrm{H}_{2} \mathrm{O} 70: 30 v / v$ solution $1 \mathrm{mg} \mathrm{mL}^{-1}$ ). A defatting with $n$-hexane was performed to completely remove the lipid fraction. The raw alcoholic extract of each sample was concentrated under reduced pressure to dryness, rinsed with $2 \mathrm{~mL}$ of extraction solvent and analyzed by HPLC with a Diode Array Detector (DAD) and HPLCl MS. The reagent gallic acid was supplied by Extrasynthèse (Lyon, France).

\section{Extraction of Phenolic Acids}

Extraction of phenolic acids was performed by a solid-phase extraction (SPE) using an Octadecyl C18 cartridge from Stepbio (Bologna, Italy). A 3-g quantity of olive oil added to $1 \mathrm{~mL}$ of resorcin solution (internal standard; diethyl ether solution $0.5 \mathrm{mg} \mathrm{mL}^{-1}$ ) was dissolved in $n$-hexane $(15 \mathrm{~mL})$ and deposited on cartridge previously washed with $2 \times 10 \mathrm{~mL}$ of methanol and $2 \times 5 \mathrm{~mL}$ of $n$-hexane. The elution steps were: $n$-hexane $(4 \times 10 \mathrm{~mL})$ at atmospheric pressure to remove lipophilic compounds, methanol $(4 \times 10 \mathrm{~mL})$ under vacuum to recover the polar fraction. After concentration under reduced pressure the fraction containing phenolic acids was rinsed with $1 \mathrm{~mL}$ of acetone and derivatized with $150 \mu \mathrm{L}$ of bis(trimethylsi1yl)trifluoracetamide (BSTFA) to perform GC analysis. The reagents resorcin and BSTFA were purchased from Merck (Darmstadt, Germany).

\section{Identification and Quantitation of Individual Polyphenols}

Identification of individual polyphenols was carried out by using data from both HPLC/DAD and HPLC/MS analyses, using their retention time, UV-vis and mass spectra. The molecular characterization of the isolated compounds, elenolic acid and deacetoxyoleuropein aglycone, was performed by ${ }^{1} \mathrm{H}$ - and ${ }^{13} \mathrm{C}$-NMR by comparison with previously reported data $[15,16]$.

The quantitative evaluation of individual polyphenols (except phenolic acids) was performed by HPLC/DAD with authentic standards, such as tyrosol, oleuropein, luteolin and apigenin which were used to prepare a four-point regression curves $\left(\mathrm{r}^{2} \geq 0.99\right)$. All these standards were purchased from Extrasynthèse (Lyon, France).

Tyrosol and hydroxytyrosol amounts were calculated at $280 \mathrm{~nm}$ using tyrosol as reference; oleuropein aglycone, deacetoxyoleuropein aglycone and other unidentified secoiridoid compounds were calculated at $280 \mathrm{~nm}$ using oleuropein as standard; phenolic acids were calculated as a sum using caffeic acid as reference at $330 \mathrm{~nm}$; elenolic acid and elenolic acid derivatives were evaluated at $240 \mathrm{~nm}$ using oleuropein as standard. For oleuropein aglycone, deacetoxyoleuropein aglycone, elenolic acid and its derivatives, the mole- 
cular weight correction was applied. The flavonoid aglycons luteolin and apigenin were evaluated by measuring the absorbance at $350 \mathrm{~nm}$ of the pure standards.

The quantity of the single phenolic acid was performed by HRGC analysis. Ferulic acid, syringic acid, caffeic acid and pcoumaric acid were purchased from Fluka (Buchs, Switzerland).

\section{Analytical Techniques and Equipment}

\section{HPLC-DADAnalysis}

HPLC/DAD analyses were performed on an HP 1090L liquid chromatograph controlled by an HP 9000 workstation (Hewlett-Packard, Palo Alto, CA, USA). The analytical column was a $4.6 \times 250 \mathrm{~mm}$ LiChrosorb RP18, $5 \mu \mathrm{m}$, (Merck) maintained at $26^{\circ} \mathrm{C}$. The HPLC/DAD analyses were performed with solvents of analytical grade purchased from Carlo Erba (Milano, Italy). Tyrosol derivatives, oleuropein, secoiridoid derivatives and flavone aglycons were analyzed according to Romani [24].

\section{HPLC-MS Analysis}

The HPLC-MS analyses were performed using an HP 1090L liquid chromatograph equipped with a DAD detector. The interface was an HP 1100 MSD APIelectrospray (Hewlett-Packard). The HPLC-MS analyses were performed according to a previous report [25].

\section{HRGCAnalysis}

HRGC was carried out on a Carlo Erba (Milano, Italy) Mega Series 5300, equipped with an on-column injection system and an FID, on a 30-m-long SPB-5 column from Supelco (Palermo, Italy); 0.32 i. d., $0.10 \mu$ film thickness. The oven temperatures were as follows: from 70 to $135^{\circ} \mathrm{C}$ at $2^{\circ} \mathrm{C} \mathrm{min}^{-1}, 10 \mathrm{~min}$ at $135^{\circ} \mathrm{C}$, from 135 to $220^{\circ} \mathrm{C}$ at $4{ }^{\circ} \mathrm{C} \mathrm{min}^{-1}, 10 \mathrm{~min}$ at $220^{\circ} \mathrm{C}$, from 220 to $270^{\circ} \mathrm{C}$ at $4{ }^{\circ} \mathrm{C} \mathrm{min}^{-1}, 20 \mathrm{~min}$ at $270^{\circ} \mathrm{C}$. The temperature of the detector was held at $280^{\circ} \mathrm{C}$ and the carrier gas was $\mathrm{He}$ at $2 \mathrm{~mL} \mathrm{~min}^{-1}$. Quantification of phenolic acids was effected by peak area integration with a Carlo Erba Mega Series Integrator.<smiles>OCCc1ccc(O)cc1</smiles>

Tyrosol<smiles>OCCc1ccc(O)c(O)c1</smiles>

Hydroxytyrosol

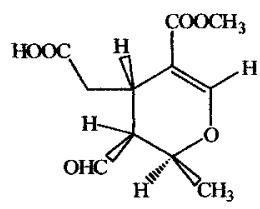

Elenolic acid<smiles>C/C=C1/C(CC(=O)OCCc2ccc(O)c(O)c2)=C(C(C)=O)COC1O</smiles>

Oleuropein aglycone<smiles>C/C=C(/C#N)C(C)(CC=O)CC(=O)OCCc1ccc(O)c(O)c1</smiles>

Deacetoxyoleuropein aglycone<smiles>C/C=C1/C(CC(=O)OCCc2ccc(O)cc2)=C(C(C)=O)COC1O</smiles>

Ligstroside aglycone<smiles>[Y]c1cc(-c2cc(=O)c3c(O)cc(O)cc3o2)ccc1O</smiles>

$\mathrm{R}=\mathrm{H} \quad$ Apigenin
$\mathrm{R}=\mathrm{OH}$ Luteolin

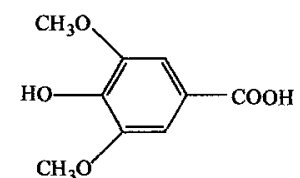

Syringic acid<smiles>O=C(O)C=Cc1ccc(O)c(P)c1</smiles>

Figure 1. Structural formulas of polyphenols and secoiridoids in olive oil.

\section{Results and Discussion}

The extraction of polyphenols and secoiridoids from olive oils and subsequent fractionation methods were performed to obtain a total recovery of these minor polar compounds.

Generally the phenolic content of an oil is obtained by a spectrophotometric evaluation of the total extract, whereas the findings of this study allow the quantification of each identified compound. To the best of our knowledge this is the first report of the quantification of deacetoxyoleuropein aglycone and elenolic acid in virgin olive oil.

The technological processes of milling, by the continuous washing with water or by press, gave two different oil samples, traditional $(\mathrm{T})$ and continuous $(\mathrm{C})$, respectively, and the compounds evaluated are reported in Table II and expressed in $\mathrm{mg} / \mathrm{L}$ of olive oil.

Two chromatographic techniques were applied: HPLC and HRGC. The first method was used to identify each compound, with the exception of the phenolic acids which were calculated as a sum and expressed as caffeic acid. The HRGC technique was more sensitive for the char- acterization of these minor constituents and allowed identification and calculation of the amounts of the single molecules.

The following compounds were identified by HPLC: hydroxytyrosol (OH-Tyr), tyrosol (Tyr), elenolic acid (EA), and two elenolic acid derivatives calculated as a sum (EA der.), deacetoxyoleuropein aglycone (DacOLagl), oleuropein aglycone, four secoiridoid derivatives calculated as a sum (Secoir. der.) and the flavones luteolin and apigenin. In this case phenolic acids were calculated as a sum and expressed as caffeic acid ( $\Sigma$ PA). Some of the structures of the characterized molecules in olive oil are presented in Figure 1.

As an example, the chromatographic profile of a Dritta oil sample, recorded at 280 and $240 \mathrm{~nm}$, is presented in Figure 2. In Figure 3 the mass spectrum of deacetoxyoleuropein aglycone is shown. The most relevant peaks, recorded at 319 and $183 \mathrm{~m} / \mathrm{z}$ corresponded to the quasi-molecular ion $[\mathrm{M}-\mathrm{H}]^{-}$and to the fragment after the loss of the 3,4-di-hydroxy-phenyl ethyl moiety. The peak at $639 \mathrm{~m} / \mathrm{z}$ correspond to the dimer of deacetoxyoleuropein aglycone.

The unidentified secoiridoid compounds showed a UV-vis spectrum similar 

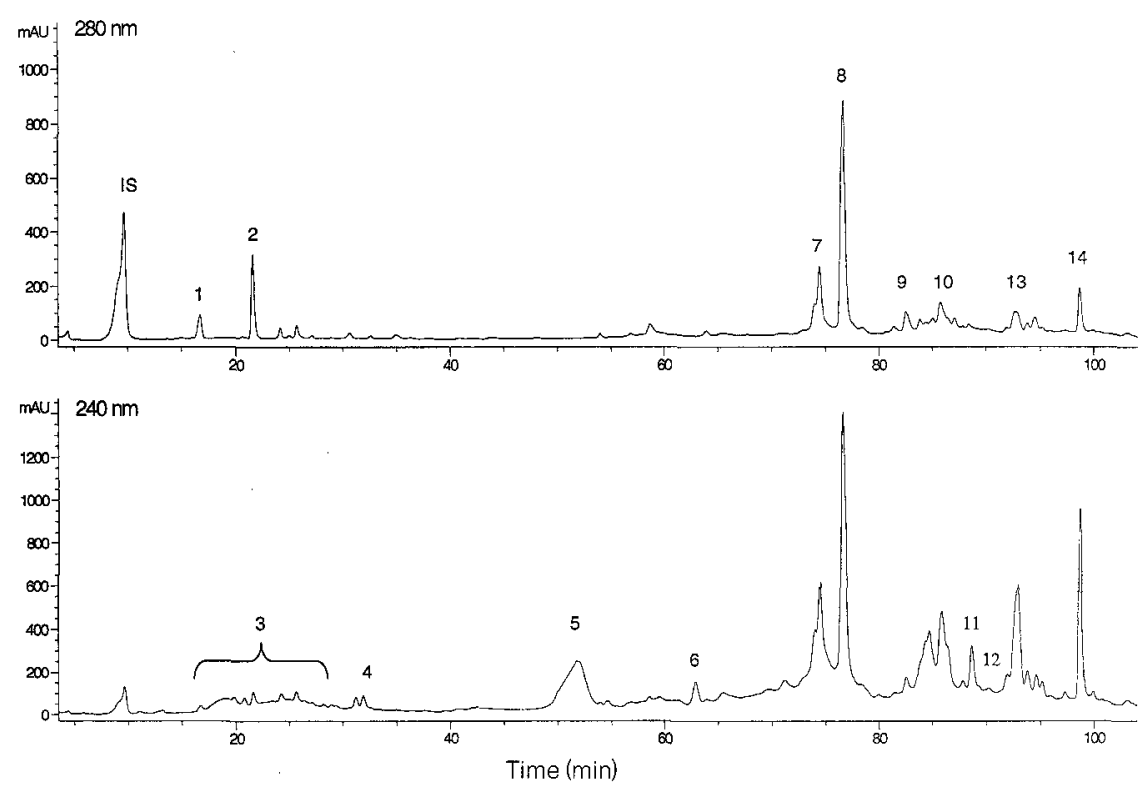

Figure 2. Chromatographic profile of olive oil extract (cv. Dritta) acquired by HPLC/DAD at 280 and $240 \mathrm{~nm}$. Eluent was $\mathrm{H}_{2} \mathrm{O}\left(\mathrm{pH} 3.2\right.$ with $\left.\mathrm{H}_{3} \mathrm{PO}_{4}\right) / \mathrm{CH}_{3} \mathrm{CN}$ (7-step linear gradient from $100 \% \mathrm{H}_{2} \mathrm{O}$ to $45 \% \mathrm{CH}_{3} \mathrm{CN}$, see also reference [24]) at a flow rate of $1.0 \mathrm{~mL} \mathrm{~min}^{-1}$ during a $106-\mathrm{min}$ run. The column was LiChrosorb RP18 $(5 \mu \mathrm{m})$ maintained at $26^{\circ} \mathrm{C}$. Peaks: IS = gallic acid; 1 = hydroxytyrosol; $\mathbf{2}=$ tyrosol; $\mathbf{3}=$ phenolic acids zone; $\mathbf{4}, \mathbf{6}=$ elenolic acid derivative; $\mathbf{5}=$ elenolic acid; $\mathbf{7}=$ deacetoxyoleuropein aglycone; $8,9,10,14=$ secoiridoid derivative; $11=$ luteolin; 12 = apigenin; $13=$ oleuropein aglycone.

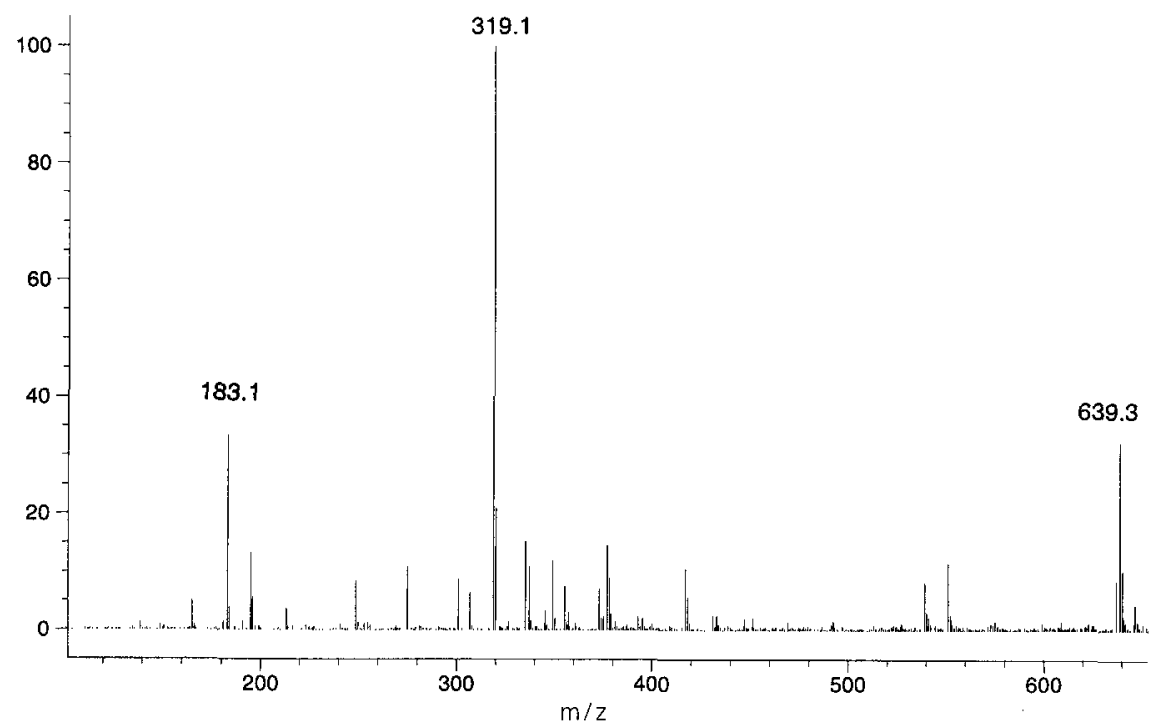

Figure 3. Negative ion mass spectrum of deacetoxyoleuropein aglycone acquired by API-electrospray HPLC-MS analysis under the following operating condition: gas $\mathrm{T} 350^{\circ} \mathrm{C}$, nitrogen flow rate $10.0 \mathrm{Lmin}^{-1}$, nebulizer pressure $40 \mathrm{psi}$, quadrupole temperature $40^{\circ} \mathrm{C}$, and capillary voltage $3500 \mathrm{eV}$. Mass spectra were recorded in the range of $0-1000 \mathrm{AMU}$.

to that of oleuropein, and the MS spectrum recorded in the negative ion mode exhibited the signal at 361 or $377 \mathrm{~m} / \mathrm{z}$, corresponding to the quasi-molecular ion of the aglycons of ligstroside and oleuropein, respectively.

The results reported in Table II indicate that some components, particularly elenolic acid and deacetoxyoleuropein aglycone, represent the main compounds in most of the samples, whereas the con- tent of luteolin and apigenin flavons is very low.

The hydroxytyrosol content is in the range of $0.75-3.75 \mathrm{mg} \mathrm{L}^{-1}$ of olive oil. This important antioxidant compound comes mainly from the hydrolysis of verbascoside, a caffeic ester present in the fruits, and from oleuropein degradation both during the fruit ripening and processing and storage of the oil. This compound could be a correlatable parameter to high values of the hydrolysis percentage (percentage of tyrosol and hydroxytyrosol of total polyphenols). It is well known that tyrosol and hydroxytyrosol concentrations are higher when the oil is aged or in advanced oxidized conditions $[17,26]$.

Concerning phenolic acids, which are an important class of antioxidant substances, a procedure to concentrate their amounts in the extract was carried out and the specific HRGC technique allowed calibration of the single compounds. In Figure 4 the chromatogram derived from a C18 SPE procedure (oil sample Gentile) reports phenolic acids, fatty acid TMS derivatives, monoglycerid TMS derivatives and secoiridoid aglycons (ligstroside and oleuropein aglycons). The indicated multiple temperature program enabled better separation of the phenolic acid and the aglycon signals.

The amounts of the main phenolic acids identified in the oils, namely ferulic, syringic, caffeic and p-coumaric acids are reported in Table III and expressed as $\mathrm{mg} \mathrm{L}^{-1}$ of olive oil.

In order to propose a more precise quantitative evaluation, the total phenolic content was calculated by adding the amounts of polyphenols and secoiridoids identified by HPLC/DAD and HPLC/MS to the phenolic acids characterized and quantified by HRGC.

As shown in Figure 5, the total amount of phenols varies from 150 to $550 \mathrm{mg} \mathrm{L}^{-1}$ among the cultivars considered. In particular, $\mathrm{G} 2 \mathrm{C}$ and $\mathrm{D} 3_{\mathrm{T}}$ show the highest values, $\quad 553.25 \mathrm{mg} \mathrm{L}^{-1}$ and $481.84 \mathrm{mg}$ $\mathrm{L}^{-1}$, respectively, whereas $\mathrm{L} 2{ }_{\mathrm{T}}$ represents the olive oil with the lowest content (149.09 $\mathrm{m} \mathrm{L} \mathrm{L}^{-1}$ ). According to the classification of Montedoro et al. [22], the analyzed oil samples have a medium-high phenolic content (cvs. Gentile and Dritta) and low amounts in the case of cv. Leccino. From the reported data it could be said that the main differences are related to the cultivar characteristics rather than the processing techniques [27].

In order to propose a specific marker for the characterization of the olive oils obtained by different cultivars and/or by using different processing techniques, the percentages of the various phenolic subclasses were calculated, as shown in Table IV.

In each sample the tyrosol $+\mathrm{OH}$-tyrosol percentage was near $2 \%$, except for $\mathrm{G} 2_{\mathrm{C}}(0.97 \%)$ and $\mathrm{L} 1_{\mathrm{C}}(5.15 \%)$. The flavon percentage is less than $2 \%$ in all the tested samples, with the exception of $\mathrm{D} 1_{\mathrm{C}}(2.7 \%)$. 
Table II. Phenolic compounds evaluated by HPLC-DAD and expressed in $\mathrm{mg} \mathrm{L}^{-1}$ of olive oil. $\Sigma$ PA = sum of phenolic acids, calibrated as caffeic acid at $330 \mathrm{~nm}$; EA der. $=$ sum of two elenolic acid derivatives, calibrated as oleuropein at $240 \mathrm{~nm}$; Secoir. Der. = sum of four secoiridoid compounds, calibrated as oleuropein at $280 \mathrm{~nm}$. Average values $\pm \mathrm{SD}$ of three olive oil samples.

\begin{tabular}{|c|c|c|c|c|c|c|c|c|}
\hline & $\mathrm{G} 1_{\mathrm{C}}$ & $\mathrm{G} 2 \mathrm{c}$ & $\mathrm{G} 3 \mathrm{~T}$ & $\mathrm{Ll}_{\mathrm{C}}$ & $\mathrm{L} 2{ }_{\mathrm{T}}$ & $\mathrm{D} 1_{\mathrm{C}}$ & $\mathrm{D} 2_{\mathrm{C}}$ & $\mathrm{D} 33_{\mathrm{T}}$ \\
\hline OH-Tyr & $3.75 \pm 0.16$ & $1.60 \pm 0.08$ & $0.75 \pm 0.05$ & $2.31 \pm 0.11$ & n. d. & $2.02 \pm 0.10$ & $2.76 \pm 0.11$ & $3.48 \pm 0.12$ \\
\hline Tyr & $5.80 \pm 0.12$ & $3.88 \pm 0.14$ & $4.05 \pm 0.20$ & $6.13 \pm 0.21$ & $4.00 \pm 0.17$ & $7.35 \pm 0.24$ & $7.86 \pm 0.22$ & $8.75 \pm 0.24$ \\
\hline$\Sigma \mathrm{PA}$ & $3.02 \pm 0.15$ & $8.17 \pm 0.28$ & $2.11 \pm 0.17$ & $5.99 \pm 0.19$ & $3.48 \pm 0.20$ & $2.04 \pm 0.10$ & $2.57 \pm 0.12$ & $4.72 \pm 0.22$ \\
\hline $\mathrm{EA}$ & $25.07 \pm 0.38$ & $14.74 \pm 0.24$ & $18.14 \pm 0.27$ & $10.41 \pm 0.20$ & $10.52 \pm 0.19$ & $43.07 \pm 0.51$ & $52.01 \pm 0.35$ & $69.48 \pm 0.74$ \\
\hline EA der. & $6.85 \pm 0.24$ & $3.32 \pm 0.13$ & $3.91 \pm 0.12$ & $6.46 \pm 0.25$ & $5.04 \pm 0.27$ & $3.42 \pm 0.15$ & $9.18 \pm 0.30$ & $22.33 \pm 0.31$ \\
\hline DAcOLagl & $31.78 \pm 0.58$ & $141.40 \pm 2.51$ & $6.21 \pm 0.23$ & $32.03 \pm 0.61$ & $1.46 \pm 0.08$ & $39.87 \pm 0.85$ & $35.02 \pm 0.91$ & $52.66 \pm 1.31$ \\
\hline OL agl. & $20.84 \pm 0.31$ & $22.04 \pm 0.32$ & $15.20 \pm 0.28$ & $5.99 \pm 0.11$ & $5.39 \pm 0.15$ & $30.92 \pm 0.62$ & $14.95 \pm 0.26$ & $11.33 \pm 0.24$ \\
\hline Secoir. der. & $232.79 \pm 3.71$ & $359.90 \pm 4.92$ & $173.82 \pm 3.04$ & $91.04 \pm 1.57$ & $120.13 \pm 1.44$ & $189.88 \pm 1.97$ & $299.09 \pm 4.11$ & $310.33 \pm 5.26$ \\
\hline Luteolin & $2.00 \pm 0.11$ & $5.58 \pm 0.17$ & $3.44 \pm 0.13$ & $1.08 \pm 0.06$ & $2.01 \pm 0.05$ & $8.58 \pm 0.20$ & $4.20 \pm 0.17$ & $3.2 \pm 0.12$ \\
\hline Apigenin & $0.25 \pm 0.01$ & $0.79 \pm 0.04$ & $0.6 \pm 0.03$ & $0.19 \pm 0.01$ & $0.54 \pm 0.02$ & $0.41 \pm 0.02$ & $0.41 \pm 0.02$ & $0.28 \pm 0.01$ \\
\hline
\end{tabular}

n. d. = not detectable.

Table III. Amounts of phenolic acids evaluated by HRGC and expressed in $\mathrm{mg} \mathrm{L}^{-1}$ of olive oil. Average values \pm SD of three olive oil samples.

\begin{tabular}{|c|c|c|c|c|c|c|c|c|}
\hline & $\mathrm{G} 1_{\mathrm{C}}$ & $\mathrm{G} 2_{\mathrm{C}}$ & $\mathrm{G} 3_{\mathrm{T}}$ & $\mathrm{Ll}_{\mathrm{C}}$ & $\mathrm{L} 2 \mathrm{~T}$ & $\mathrm{D} 1_{\mathrm{C}}$ & $\mathrm{D} 2_{\mathrm{C}}$ & D3 ${ }_{\mathrm{T}}$ \\
\hline Ferulic ac. & $1.52 \pm 0.12$ & $0.84 \pm 0.04$ & $0.41 \pm 0.03$ & $3.04 \pm 0.17$ & $2.20 \pm 0.16$ & $0.46 \pm 0.01$ & $1.18 \pm 0.10$ & $1.01 \pm 0.03$ \\
\hline Syringic ac. & $0.86 \pm 0.09$ & $7.61 \pm 0.15$ & $1.66 \pm 0.08$ & $2.25 \pm 0.11$ & $1.21 \pm 0.11$ & $0.74 \pm 0.07$ & $1.38 \pm 0.12$ & $4.50 \pm 0.19$ \\
\hline Caffeic ac. & $0.63 \pm 0.05$ & $1.07 \pm 0.03$ & $0.47 \pm 0.05$ & $0.63 \pm 0.03$ & $0.63 \pm 0.02$ & $0.47 \pm 0.03$ & $0.47 \pm 0.02$ & $0.47 \pm 0.02$ \\
\hline p-Coumaric ac. & $1.57 \pm 0.14$ & $2.00 \pm 0.12$ & $0.71 \pm 0.02$ & $2.29 \pm 0.13$ & $1.00 \pm 0.08$ & $1.57 \pm 0.11$ & $0.86 \pm 0.05$ & $0.57 \pm 0.02$ \\
\hline
\end{tabular}

Table IV. Percentage of the different phenolic subclasses in each oil sample. $\Sigma P^{\prime} A^{\prime}=$ sum of phenolic acids quantified by HRGC; EA der. Tot. = EA + EA der.; Secoir. Der. Tot. = DAcOLagl + OL agl. + Secoir. der.; Flavons $=$ Luteolin + Apigenin.

\begin{tabular}{|lcccccccc|}
\hline & $\mathrm{G}_{\mathrm{C}}$ & $\mathrm{G} 2_{\mathrm{C}}$ & $\mathrm{G}_{\mathrm{T}}$ & $\mathrm{L}_{\mathrm{C}}$ & $\mathrm{L}_{\mathrm{T}}$ & $\mathrm{D} 1_{\mathrm{C}}$ & $\mathrm{D}_{\mathrm{C}}$ & $\mathrm{D}_{\mathrm{T}}$ \\
\hline OH-Tyr + Tyr & 2.86 & 0.97 & 2.09 & 5.15 & 2.59 & 2.85 & 2.81 & 2.50 \\
LPA & 1.30 & 2.03 & 1.42 & 5.01 & 3.27 & 0.98 & 1.03 & 1.34 \\
EA der. Tot. & 9.58 & 3.19 & 9.61 & 10.29 & 10.09 & 14.14 & 2.43 & 18.79 \\
Secoir. Der. Tot. & 85.6 & 92.6 & 85.1 & 78.8 & 82.4 & 79.3 & 92.5 & 76.6 \\
Flavons & 0.67 & 1.13 & 1.76 & 0.77 & 1.65 & 8.99 & 1.22 & 0.71 \\
\hline
\end{tabular}

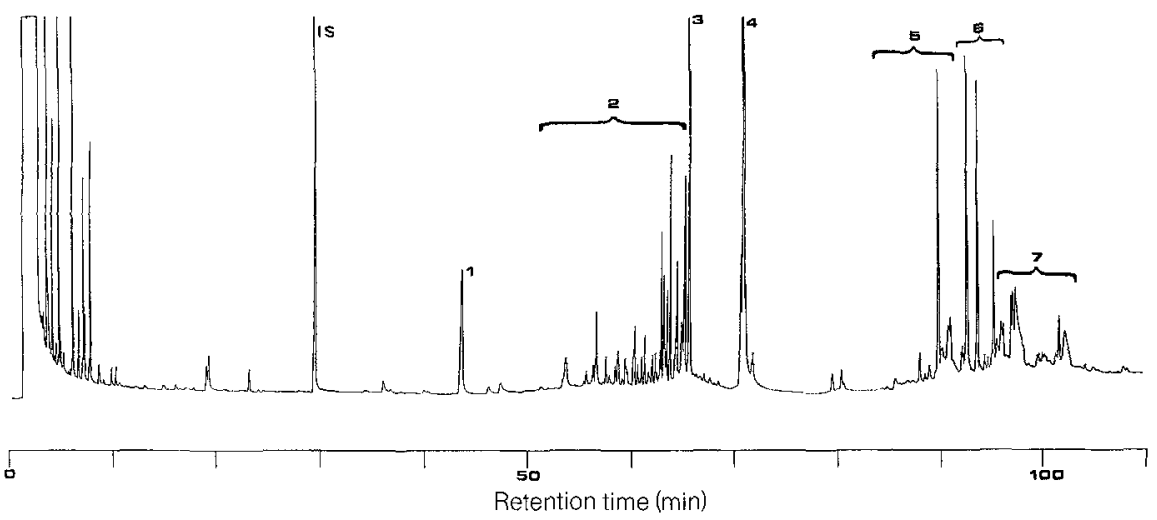

Figure 4. Chromatographic profile acquired by HRGC of an oil sample (cv. Gentile) obtained from the C18 LSE procedure. The oven temperatures were as follows: from 70 to $135^{\circ} \mathrm{C}$ at $2{ }^{\circ} \mathrm{C}$ min $^{-1}$, $10 \mathrm{~min}$ at $135^{\circ} \mathrm{C}$, from 135 to $220^{\circ} \mathrm{C}$ at $4^{\circ} \mathrm{C} \mathrm{min}^{-1}, 10$ min at $220^{\circ} \mathrm{C}$, from 220 to $270^{\circ} \mathrm{C}$ at $4^{\circ} \mathrm{C} \mathrm{min}^{-1}, 20 \mathrm{~min}$ at $270^{\circ} \mathrm{C}$. The temperature of the detector was held at $280^{\circ} \mathrm{C}$ and the carrier gas

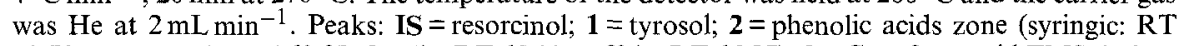
59.78; p-coumaric: RT 60.87; ferulic: RT 65.33; caffeic: RT 66.87); $\mathbf{3}=\mathrm{C}_{16: 0}$ fatty acid TMS derivative; $\mathbf{4}=\mathrm{C}_{18: 0}, \mathrm{C}_{18: 1}$ and $\mathrm{C}_{18: 2}$ fatty acids TMS derivatives; $\mathbf{5}=$ ligstroside aglycons zone; $\mathbf{6}=$ monoglycerid TMS derivatives zone; $7=$ oleuropein aglycons zone.

The phenolic acids represent less than $3 \%$ in all samples, except for $\mathrm{L} 1_{\mathrm{C}}(5.01 \%)$ and $\mathrm{L} 2 \mathrm{~T}(3.27 \%)$.

Deacetoxyoleuropein aglycone and oleuropein aglycone and the secoiridoid derivatives are the main olive oil compo- nents, their content being nearly or more than $80 \%$ of the total in the all samples. Further studies are in progress to isolate the unknown secoiridoid derivatives and to identify the possible sources of hydroxytyrosol, an important substance from a

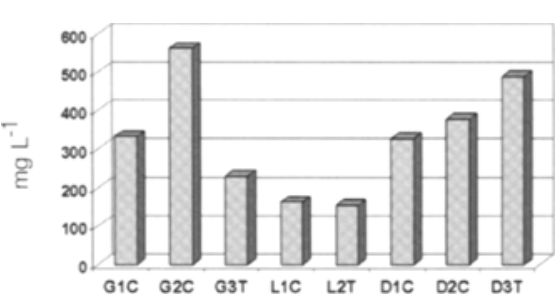

Figure 5. Total phenolic content in different Abruzzo's olive oil, from processing the fruits by continuous washing with water $(\mathrm{C})$ or by traditional press $(T)$. The total concentration was calculated by adding the amounts of polyphenols and secoiridoids identified and quantified by HPLC-DAD to the phenolic acids characterized and quantified by HRGC. Quantitative data are expressed as $\mathrm{mg} \mathrm{L}^{-1}$ of olive oil.

nutritional point of view and for olive oil stability.

In addition, it must observed that high amounts of oleuropein derivatives are associated with smaller quantities of elenolic acid and derivatives; these results, particularly evident in Dritta oil samples, could represent an interesting tool for investigating olive oil stability (Table IV).

Phenols are closely related to the quality of virgin olive oil, both for their contribution to nutrition and to oil stability. Data reported in the present work could be useful for better correlating nutritional characteristics of olive oil to its chemical pattern. In our opinion there is an urgent need for an in-depth quali-quantitative analysis of polyphenols and secoiridoids in olive oil. In order to attain this goal, evaluation of either the single compounds or the different subclasses is appropriate. 
This approach, rather than the quantification of the total phenolic compounds (expressed as different standards) by using analytical methods that cannot be compared, could give as yet unknown information.

\section{References}

[1] Navarro, M.D.; Periago, J.L.; Pita, M.L.; Hortelano P., Lipids, 1994, 29, 845-848.

[2] Perrin, J.T.; Corps Gras, 1992, 39(1/2), $25-32$.

[3] Ciappellano, S.; Simonetti, P.; Brighenti, F.; Bermano G.; Testolin G. Grasas y Aceites 1994, 45(1-2), 48-52.

[4] Wiseman, S.A.; Mathot de Fouw, N.J.; Tijburg, L.B. Atherosclerosis 1996, $120(1-2), 15-23$

[5] Visioli, F.; Galli C. Nutrion Reviews 1998 $56(5), 142-147$.

[6] Visioli, F.; Romani, A.; Mulinacci, N.; Zarini, S.; Conte, D.; Vincieri, F.F.; Galli, C. J. Agric. Food Chem. 1999, 47, $3397-$ 3401.

[7] Driss, F.; Duranthon, V.; Viard, V. Corps Gras 1996, 3(6), 448-451.
[8] Amiot, M.J.; Fleuriet, A.; Macheix, J.J. J. Agric. Food Chem. 1986, 34, 823-826.

[9] Ranalli, A.; De Mattia, G.; Ferrante, M.L.; Giansante, L. La Rivista delle Sostanze grasse 1997, 74, 501-505.

[10] Tayfun Agar, I.; Hess-Pierce, B.; Sourour, M.M.; Kader, A.A. J. Agric. Food Chem. $1998,46,3415-3421$.

[11] Garcia, J.M.; Gutierrez, F.; Castellano, J.M.; Perdiguero, S.; Morilla, A.; Albi, M.A. J. Agric. Food Chem. 1996, 44, 264267.

[12] Montedoro, G.; Garofolo, L. La Rivista Italiana delle Sostanze Grasse 1984, 61, 157-168.

[13] Solinas, M.; Cichelli, A. Riv. Ital. Sci. Aliment. 1982, 11, 223-230.

[14] Cichelli, A.; Solinas, M. Riv. Merceol. 1984, 23, 55-58.

[15] Gariboldi, P.; Jommi, G.; Verotta, L. Phytochemistry 1986, 25(4), 865-869.

[16] Lo Scalzo, R.; Scarpati, M.L. J. Nat. Prod. 1993, 56, 621-623.

[17] Montedoro, G.; Servili, M.; Baldioli, M.; Selvaggini, R.; Miniati, E.; Macchioli, A. J. Agric. Food Chem. 1993, 41, 2228 2234.

[18] Litridou, M.; Linsen, J.; Schols, H.; Bergmans, R.; Posthumus, M.; Tsimidou, M.; Boskou, D. J. Sci. Food Agric. 1997, 74, 169-174.
[19] Romani, A.; Mulinacci, N.; Pinelli, P.; Vincieri, F.F.; Cimato, A. J. Agric. Food Chem. 1999, 47, 964-967.

[20] Pirisi, F.M.; Cabras, P.; Falqui Cao, C. Migliorini, M.; Muggelli M. J. Agric. Food Chem. 2000, 48(4), 1191-1196.

[21] Damtoft, S.; Franzyk, H,; Rosendal Jensen, S. Phytochemistry 1992, 31(12), 4197-4201.

[22] Montedoro, G.; Servili, M.; Baldioli, M. Miniati E. J. Agric. Food Chem. 1992, 40(9), 1577-1580.

[23] Montedoro, G.; Servili, M.; Baldioli, M.; Miniati, E. J. Agric. Food Chem. 1992, $40(9), 1571-1576$

[24] Romani, A.; Baldi, A.; Mulinacci, N.; Vincieri, F.F.; Tattini, M. Chromatographio 1996, 42 (9/10), 571-577.

[25] Romani, A.; Pinelli, P.; Mulinacci, N.; Vincieri, F.F.; Tattini, M. Chromatographia 1999, $49(1 / 2), 17-20$.

[26] Cioni, F.; Modi, G.; Siminani, G.; Tracchi, S. Boll. Chim. Igien. 1998, 49, 115118.

[27] Servili, M.; Baldioli, M.; Montedoro, G.F. Acta Hortic. 1994, 356, 331-334.

Received: Jul 25, 2000 Revised manuscript received: Oct 16, 2000 Accepted: Nov 1, 2000 